\title{
Bi-Directional Drone Design and its Path Planning
}

\author{
Soutrik Mukherjee
}

\begin{abstract}
There are two aspects to my project, one is optimized bi-directional drone designing and other is its path planning. The optimization in design lies in the fact that my drone can carry load both in $+z$ and $-\mathrm{z}$ axis if its direction of motion is in $x-y$ plane. This design optimization helps the drone to carry more payload than drones of same frame(basic chassis) weight category. In other words, my drone has greater payload to its own weight ratio(almost 0.8 ) than other drones with almost similar or same functionalities. Coming to path planning algorithm, I have taken a mathematical induction approach to solve the problem statement by clearly defining our conditions to follow to remain in the specified path along with constraints lying in the path. My goal as a path planner has to ensure that the drone follows the conditions specified without grappling into obstacles. Also, to achieve the desired goal in least possible time. The paths traversed by the drone would be stored into the memory processing system of the drone for future development of algorithm.
\end{abstract}

Keywords: Bi-directional Drone, Design Optimization, Payload to Weight ratio, Path-planning, Mathematical Induction

\section{INTRODUCTION}

$\mathrm{D}_{\mathrm{r}}$ ones having its enormous sea of potentials, perhaps known to popular culture through its use in all-time great movies like- James Bond Sky Fall, Wolf of Wall Street, Interstellar and Iron Man 2 opens a new genre of technology by harnessing the force of nature.

\section{A. HISTORY OF DRONE TECHNOLOGY}

Drones were popularized due to its massive potential in war, making appearances in the modern era as a surveillance tool during World War-II. Israel Air Force showed that there can be other applications of drone along with surveillance, inculcating weaponry and modern battlefield tactics into the functionalities of drone and hence making an unmanned aerial machine that could be an addition to the military force of the army[4]. Slowly it sipped into the military forces of US and some other countries. Later in 2010s, these drones were easily accessible to almost any nations around the world. 2015 saw a massive increase in its funding due the spreading of technology into the popular market[4]. It was used in filmography, photography, scientific observations, architecture industry, automotive sector, inventory management aside from its application in military and surveillance.

Manuscript received on August 21, 2021.

Revised Manuscript received on August 30, 2021.

Manuscript published on September 30, 2021.

*Correspondence Author Industrial Design, National Institute of Technology, Rourkela, India Email: soutrik.viratech@gmail.com

(c) The Authors. Published by Blue Eyes Intelligence Engineering and Sciences Publication (BEIESP). This is an open access article under the CC BY-NC-ND license (http://creativecommons.org/licenses/by-nc-nd/4.0/)
Soutrik Mukherjee*, Undergraduate Researcher, Department of

\section{B. RECENT TREND}

Recently, the rapid development of Artificial Intelligence and Machine Learning tools have been infused into the earlier man controlled drone producing autonomous self-developing drones. The drone that I have made can take an overall impression of a situation including motion, speed, obstacle density, wind speed and using these data it can self-learn best possible trajectory with least possible obstacle to have a better rate of success in subsequent trials.

\section{PROSPECTIVE FUTURE}

Though the rate of development of narrow AI is very slow but in the future it is not absurd to expect a fully autonomous intelligent drone system. With my limited knowledge I believe that, the probability of drone gaining intelligence to the level that it may harm our civilization is extremely small though a regulatory government system keeping in track its progress may be a future possibility

\section{MOTIVATION}

Looking into the trends in technology there are these paradigm shifts in technology, a particular kind of technology defining the era, creating an awe-inspiring effect on collective humanity, harnessing the force of nature into the best possible way, and obviously defining the way market should be operated in the near future

Like this, the next paradigm shift in my opinion would be drone technologies. If we investigate the estimated investment in drone hardware there is a rather linear increase from 2015, however one thing to keep in mind is- in an exponential curve there is always an initial linear phase. I think we are looking into the initial linear growth of a future exponential rise in drone technologies.

The first drone was developed in 1917 named Ruston Proctor Aerial Target which became the first pilotless winged aircraft in history. Then the excitement stagnated, and funding reduced for a long time due to sheer lack of belief among people on this way ahead of time pilotless flight technology. Again, in 2010s, it was popularized for having civilian applications. After that there was always a rise in generation of interest going proportionally with funding, among entrepreneurs and technologists. It came into popular market in 2014s gathering more scope in 2015, was used for various application like CCTV surveillance, architecture, automotive industry, photography, filmmaking and there was an exponential gain of investment in this particular Technology[1].Recently, intelligent drone(the drone which can take data from the surrounding and respond to its surroundings) is the future. Hence my attempt to make an intelligent bi-directional drone system[2]

Published By:

Blue Eyes Intelligence Engineering and Sciences Publication (BEIESP)

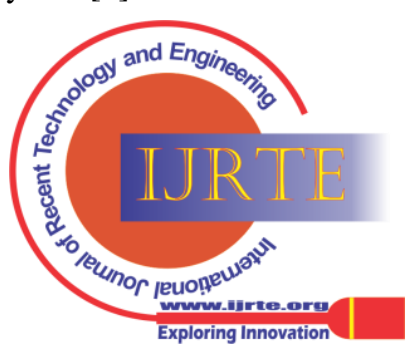




\section{Bi-Directional Drone Design and its Path Planning}

\section{LITERATURE REVIEW}

As there are two parts to my project, one is the design optimization aspect, another would be the path planning aspect. Keeping the inherent differences of both these topics in mind literature review is divided into two parts

\section{A. Designing and Conceptualization}

Drone sizes vary from vast fixed-wing UAV, with a wing span of $61 \mathrm{~m}$ and a weight of $15,000 \mathrm{~kg}$, to smart dust (SD) which is composed of several micro-electro-mechanical systems, with a minimum size of $1 \mathrm{~mm}$ and a weight of 0.005 g (Hassanalian and Abdelkefi (2017)). Such a wide range of physical features give us the scope to design a drone matching with the kind of requirements we want the drone to have. A high payload to its frame weight ratio is always a feature that is welcomed in the designing iterations of a drone. Some other design developments are done on the weight by Arjomandi et al.(2006) and Weibel and Hansman (2006) who both identify five categories, whereas Singhal et al. (2018) divide drones into 10 classes

\section{B. Path Planning}

The concept of distance transformation was first proposed on a binary image by Rosenfeld and Pfaltz. Distance transformation is used to convert a digital binary image that consists of object (foreground) and non-object (background) pixels into another image, in which each background pixel has a value corresponding to the minimum distance from the object by a distance function(Fangyi Li et. al). Later on problems regarding vehicle routing were discussed by Daniela Rojas Viloria et. al..

\section{Objective Statement}

All these papers along with references from google and books on both designing and path planning (Principles of Robot Motion: Theory, Algorithms, and Implementations), I have realized that basic problems that I wanted to work upon are increasing the payload to frame weight ratio and providing autonomy to the functioning of the drone

\section{PROPOSED WORK}

The basic design procedure of the application to be developed was preceded by the studying various related designs for the particular functionality that is targeted to achieve. It is interesting to note that the basic constraint between making the physics of drone work as well as adding an aesthetic and structural integrity to the design was a key challenge while designing and path planning of the systems and sub-systems.

\section{A. Functionalities}

a) Utilities of the Robot:-

1) Autonomously sense and detect obstacles in all 6 directions

2) A slightly over 180 degree forward-facing horizontal arc of their visual field

\section{b) Activities it can perform:-}

1) Beyond the basic requirement-

i) Infrastructure development: - Obstacle Detection Sensor (6 directional) along with autonomous bi-directional weight carrying ability endows it with the capacity to produce a pre-programmed structural system with more than $95 \%$ accuracy

ii) Inventory Management: - From raw material discovery to assemble line inspection, my drone may take on tasks too difficult for large, pre-programmed robots and too dangerous or intricate for humans.

iii) Surveillance and security: - With real-time accurate aerial data, responders will be able to make the best decision to handle hostile situations effectively and safely.

2) Basic Requirement-

i) Payload Carriage: - It can carry a payload of around 6 Kg(above and below combined) throughout an indoor or outdoor system with a fairly high self-learning obstacle detection ability

\section{c) Out of the box functionalities: -}

1) Bi-Directional Load carrying ability

2) High Payload to weight ratio(almost 0.8)

\section{B. Design Specification}

\section{a) Material Specifications:-}

1) Frame- Keeping the most important feature of frame as strength we used thermoplastics such as variants of nylon, polyester, and polystyrene, because they are inexpensive to make into complex parts using injection molding processes[1]. Another preferred choice would be carbon fiber-reinforced composites. These composites offer high strength, low density, and high

stiffness to make rigid drone frames[4]

2) Motors- Conventional electric motors are used with Copper windings and permanent magnet systems. The housing of the motors were chosen to minimize weight, and either thermoplastics or aluminum alloys present good strength-to-weight ratios[1]. Motors can generate significant heat. So, materials with high thermal conductivity, like aluminum, can be used for the housing to help cool the motor.

3) Propellers- The rotor blades of drones turn at high speeds, so they tend to absorb the most wear-and-tear when a drone flies. Rotor blades would be made from carbon fiber-reinforced composites[1]. However, rotor blades are frequently damaged and replaced, so another preferred choice may be thermoplastics to reduce the cost of replacing them when they break.

4) Batteries- Lithium-Ion batteries is the preferred choice for best Power to Weight ratio[1]

\section{b) Sensors Used: -}

1) Obstacle Avoidance and Autopilot Sensors- Drone on autopilot can fly a programmed route, called waypoints and take aerial photos or video much better than any pilot can with an obstacle detection capacity of about 10 times more than a humanoid pilot

2) Tilt sensors- They are tied into feedback loops with the drone's motors to estimate the required tilt in a trajectory

3) Current and voltage sensors- They help the drone track the energy drawn from its power reserves, helping its pilot know when it is time to land and recharge.

4) GPS and magnetic sensors- Aids in navigation by measuring the drone's location and orientation[6].

Published By:

Blue Eyes Intelligence Engineering and Sciences Publication (BEIESP) 
5) Airflow sensors- Allow drones to detect their airspeed or wind currents, and that information can be fed back into its balancing circuits to make the drone's flight even more stable.

6) Microchip technology- Inculcates almost 180-degree smart vision

\section{Drone Visualizations}

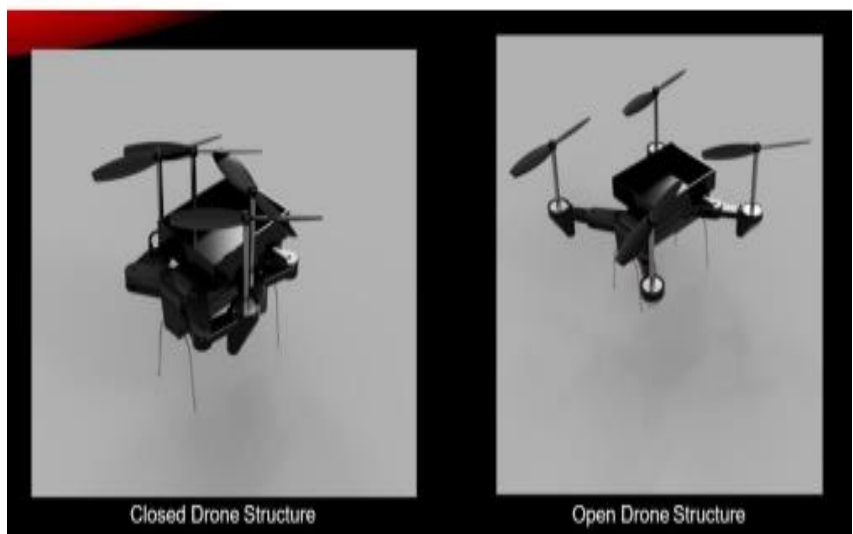

Fig.1

\section{Labelled 3-D View}

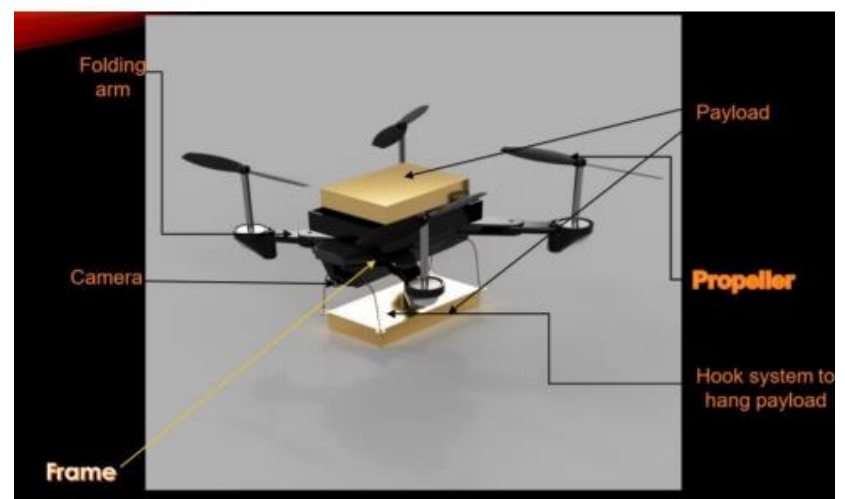

Fig.2

\section{Kind of wavsin which loadine can be done}

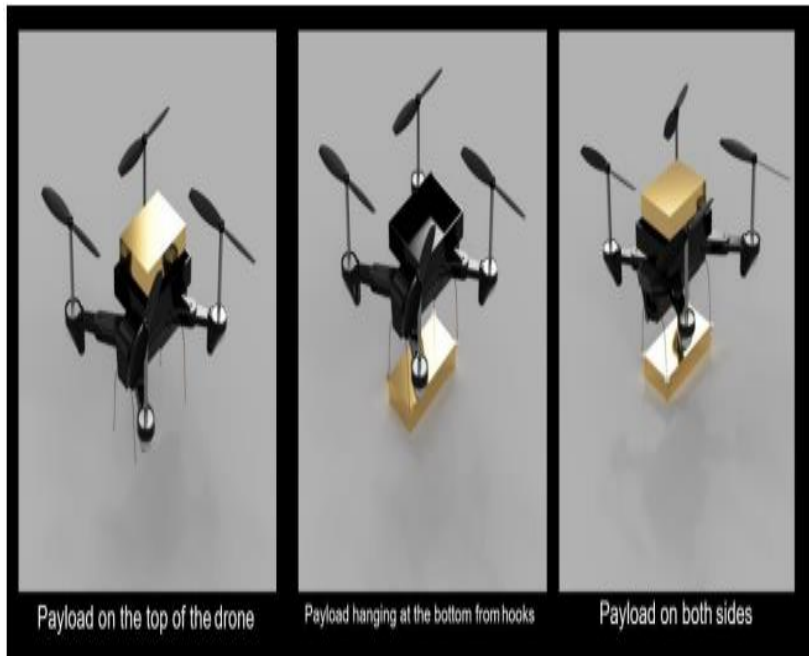

Fig.3

\section{Path Planning}

A mathematical induction approach to do path planning is done in the drone, in which I have defined conditions to follow and the constraints that the drone would have while following those conditions. My goal as a path planner would be to ensure that the instantaneous co-ordinate of the drone is never equal to the instantaneous co-ordinate of the obstacle because if that case happens then our drone will collide with the obstacle at that particular point[5].

\section{a) Variables used: -}

\section{Å-Initial State}

Å-Initial State

A-Location at any time $t$

$f\left(x_{\text {initial }}, y_{\text {initial }}, Z_{\text {initial }}\right)$ - Function defining the initial state

$\mathrm{g}\left(\mathrm{x}_{\text {final }}, \mathrm{y}_{\text {final }}, \mathrm{z}_{\text {final }}\right)-$ Function defining the final state

$\mathrm{u}$ is the function containing the obstacle in the space during the time interval $\mathrm{T}$

This explains the condition: - $A \neq u, \forall(u, A) € \circledR$

\section{b) Problem Statement: -}

\section{Conditions: -}

Initial State, $\AA \sim \mathrm{f}\left(\mathrm{x}_{\text {initial }}, \mathrm{y}_{\text {initial }}, \mathrm{Z}_{\text {initial }}\right)$

Final State, $\mathrm{A} \sim \mathrm{g}\left(\mathrm{x}_{\text {final }}, \mathrm{y}_{\text {final }}, \mathrm{Z}_{\text {final }}\right)$

Constraints: -

$u:[f \gg g, T]$

Where $\mathrm{u}$ is the function containing the obstacle in the space during the time interval $\mathrm{T}$

\section{Goal: -}

To reach from $\AA$ A to À such that its instantaneous state $\mathrm{A} \neq \mathrm{u}, \forall(\mathrm{u}, \mathrm{A}) € ®$ in the time interval $\mathrm{T}$

\section{Assumption: -}

This is a scenario with negligible effect of wind on the efficiency of drone

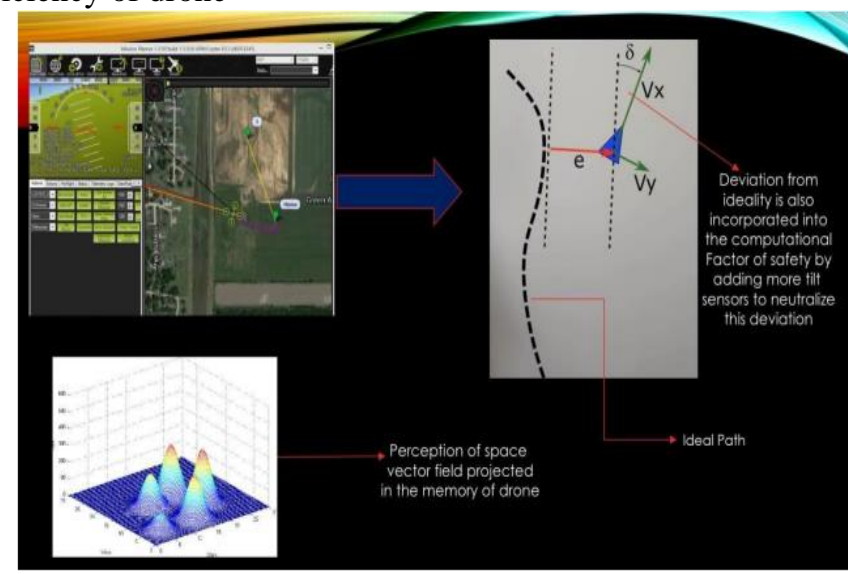

The deviation $\delta$ happens because in the algorithm we have assumed that there is negligible effect of wind but in reality there is some effect of wind. The assumption is taken to reduce computational complexity, but later on computational factor of safety and tilt is introduced into the drone to negate this assumption as much as possible.

Fig.4

telling the drone to takeoff into the air to a certain altitude or moving from one GPS waypoint to another GPS waypoint or commanding the drone to move forward.):- Python based (DroneKit).

\section{F. Limitations of the drone}

Every invention has some limitations in it. The work of an engineer or a designer also lies in finding those limitations along with giving functionalities 


\section{Bi-Directional Drone Design and its Path Planning}

a)

Weak GPS technology:- Because GPS does not work inside most buildings, drone sometimes cannot be used effectively indoors.

Lack of narrow AI:- The slow rate of learning from the visual database presented through surroundings make the technology to be fully self-aware a future possibility and not a present reality

Effect of wind:- Fans cause airflow and drafts. These disturbances in the air vary a lot and causes the drone to give greater deviations than expected

Inconsistent indoor vs. outdoor tech: - Right now, drones operating in an outdoors are set up with RFID tags for testing. While indoors drones are using either an optical scanner for barcodes or a camera that recognizes a number pattern for inventory management. With the pros and cons of both these methods still in testing, there's no clear option for the best technology to use inside

\section{E. Brief on Programming Modules}

Programming Language to be used: -

1) Firmware Cases: - (Communication with the hardware being used and provides the drone with its basic functionality. Things like calling measurement readings from IMUs(Inertial Measurement Units), which act as algorithmic inputs to determining what PWM(Pulse-Width-Modulation) values should be sent to the motors to keep the drone level Or reading incoming bytes from a telemetry[7]/radio receiver and writing how the incoming bytes should be parsed Or constantly reading the battery levels and trigger a buzzer if the voltage gets below a certain level[5]):- $\mathrm{C}$ and $\mathrm{C}++$ based(ArduPilot, PX4)

2) Application Cases: - (High level workings of the drone, where we treat our drone as a magical black box/API that reliably responds to commands we send to it[5]. Things like

\section{G. Business Angle and Future Scope}

Initially, to produce high utility, having high application case drones can be produced to create a brand value and image. Those drones may have applications in military, weaponry and performing complex tasks[1]. If the product sells then the market can be spread with medium cost, medium utility drones having its use in filmography, photography, surveillance and later to produce mass drones having low cost and low utilities which may be used for recreational purposes An approach would be: -

\section{-a) High cost, High Utility}

drones- For selected sections

of industry,

- b) Medium Cost, Medium Utility Drones- For more varied sections of industry,

-c) Low Cost, Low Utility Drones- For mass customized industrial benefits.

However, finding the best approach would require solving a lot of logistics and business challenges

\section{CONCLUSION}

Drones are becoming commonplace in both the commercial and non-profits sectors. In the near future their

use will be even more widespread. Through this work, I wanted to optimize the effectively of current drone to improve weight carrying ability.

There has been innumerable development recently in the field AI and ML, equating all these factors autonomous drone seems to be the way to future. As a technology enthusiast, I always wanted to work on exciting engineering problems with unabridged dedication and hard work hence was my decision to choose this topic. I believe I have shown the motivation and most importantly the tenacious interest in pursuing some of these complex issues.

Lastly the main aim of this project was to improve myself and try to uphold the general charm of scientific thinking to the fullest of my ability.

\section{REFERENCES}

1. Classifications, applications, and design challenges of drones: A review"(2017) (lM.Hassanalian, A.Abdelkefi)

2. CLASSIFICATION OF UNMANNED AERIAL VEHICLES" (2006) (Maziar Arjomandi, Shane Agostino, Matthew Mammone, Matthieu Nelson,Tong Zhou)

3. Safety Considerations for Operation of Unmanned Aerial Vehicles in the National Airspace System”(2006) (Weibel, Roland E; Hansman, R. John)

4. Unmanned Aerial Vehicle Classification, Applications and Challenges: A Review"(2018) (Gaurav Singhal, Babankumar shyam Bansod, Lini Mathew)

5. Distance functions on digital pictures" (1968) (A.Rosenfeld, J.L.Pfaltz)

6. 3D-visualization ablation planning system assisted microwave for

7. hepatocellular carcinoma (Diameter >3): a precise clinical application"(2020) (Chao An et. al)

8. Path Planning for UAV to Collect Sensors Data Based on Spiral

9. Decomposition"(2018) (Wu Yue, Z.Jiang)

\section{AUTHORS PROFILE}

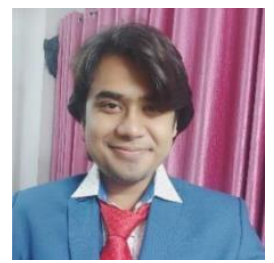

Soutrik Mukherjee, I'm currently in my final year of undergraduate engineering curriculum at NIT Rourkela, India, majoring in Industrial Design and minoring in Mechanical Engineering. have received several accolades for my academic endeavors including the medal of academic excellence for securing 1st position in my department. I have been selected from a pool of vast applicants as a Summer Research Fellow(SRF) in the coveted Indian Academy of Sciences, Bangalore where my work involved "Algorithms to estimate Laser Keyhole Welding profile". I also had the golden opportunity to intern at IIST(Indian Institute of Space Science and Technology), the space-tech wing of ISRO, on "Numerical Studies of Laminar Fluid Flow". Currently I'm working as an undergrad researcher at IIT Kanpur on designing and modal analysis of an experimental set-up for bubble drop coalescence. My scope of interest includes- Vibration and Control, Structural Analysis, Fluid Mechanics and Flows, Heat Transfer, Product Design

Published By:

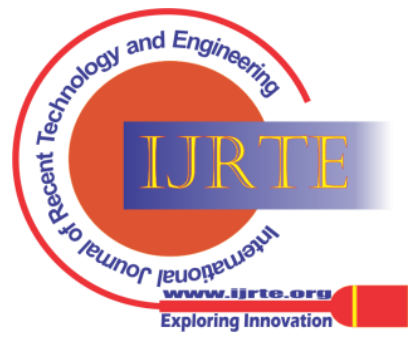

